\title{
NOVAS SINONÍMIAS E CORREÇÕES EM SIMULIUM COM A REVALIDAÇÃO DE S. PRUINOSUM LUTZ, 1904 (CULICOMORPHA, SUMULIIDAE)*
}

\author{
Victor Py-Daniel**
}

\begin{abstract}
PY-DANIEL, V. Novas sinonímias e correçðes em Simulium com a revalidação de $S$. pruinosum Lutz, 1904 (Culicomorpha, Simuliidae). Rev. Saúde públ., S. Paulo, 23:254-7, 1989.

RESUMO: Consideram-se Simulium antunesi (Lane \& Porto, 1940), S. mauense Nunes de Mello, 1974 e S. nilesi Rambajam, 1979 sinonimias de Simulium perflavum Roubaud, 1906; S. sucamense Nunes de Mello, 1974 e S. santaelenae Ramirez-Pèrez \& Peterson, 1981 sinonimias de S. (Psilopelmia) iracouboense Floch \& Abonnenc, 1946; S. major Lane \& Porto, 1940 sinonimia S. (Helmicnetha) rubrithorax Lutz, 1909. Ressalta-se a utilizaçăo errônea do nome Grenierella para o subgênero Grenieriella Vargas \& Nájera, 1951. Simulium (G.) pruinosum Lutz, 1910 é revalidada, e sugere-se que S. nigrimanum Macquart, 1838 volte a ocupar a plosiçào de species inquirendae.
\end{abstract}

DESCRITORES: Simuliidae, classıfıcaçăo.

Simulium perflavum Roubaud, 1906

Simulium antunesi (Lane \& Porto', 1940) syn.n.

Simulium mauense Nunes de Mello ${ }^{10}, 1974$ syn.n.

Simulium nilesi Rambajan", 1979 syn.n.

Após examinar o material-tipo de S. antunesi, $S$. mauense e coletar material em toda a fronteira Brasil/Guiana (localidades originais de $S$. mauense e de $S$. nilesi), não consegui reconhecer caracteres que possam separar estas três espécies de Simulium perflavum.

Portanto, proponho integrem a sinonímia desta última.

Material examinado: Simulium antunesi (Lane \& Porto $\left.{ }^{7}, 1940\right)$ FSP: n? 1323 (1 fêmea, em alf., Holótipo), n: $1264<=5275>$ (1 fêmea, em lam., Parátipo), n? 1265 (1 fêmea, em alf., Parátipo). Material coletado em Pirajá, Bahia, Brasil, por P.C.A. Antunes, 13.VI.1935. Simulium mauense Nunes de Mello ${ }^{10}$, 1974, INPA: n. 5947-19A (1 pupa, no alc., fêmea perdida, Holótipo), n? 5947-14 (1 macho, alf. e 1 exúvia pupal no alc., Alótipo), n. 5947-8 (1 macho, alf. e exúvia pupal no alc., Parátipo), n. 5947-9 (1 fêmea, alf. e exúvia pupal no alc., Parátipo), n.'s 5947-1, 5947-3, 5947-17, 5947-20 e 5947-21 (machos em alf., com exúvias pupais no álcool
$=$ material adicional), n:s 5947-2, 5947-10, 5947-16, 5947-23 e 5947-24, (fêmeas em alf., com exúvias pupais no álcool = material adicional), no 5947 (larvas e pupas no álcool = material adicional). Material coletado no Igarapé Háu-Háu, a $1 \mathrm{Km}$ de Conceição do Maú (local originalmente não nominado), por Nunes de Mello \& Eduardo V. da Silva, XII/1972. INPA n. 6199 (muitas larvas e pupas no álcool), n.s 6199-1, 6199-6A, 6199-8, 6199-9 (machos em alf., com as respectivas exúvias pupais no álcool), $n$.s 6199-2A/2B/2C, 6199-6B, 6199-15 a 6199-19 (machos e respectivas exúvias pupais no álcool), n.s $6199-7$ (macho em lam.), nis 6199-4, 6199-6C, 6199-10 a 14 (fêmeas em alf.), n.'s 6199-2D (fêmeas no álcool), n.s 61995A/5B (fêmeas em lam.), topótipos, V. PyDaniel, 11/X/1978.

Simulium (Psilopelmia) iracouboense Floch \& Abonnenc, 1946

Simulium sucamense Nunes de Mello, 1974 syn.n.

Simulium sanıaelenae Ramirez-Pérez \& Peterson, 1981 syn.n.

Ramírez-Pérez ${ }^{12.13}$ (1983, 1987), Crosskey ${ }^{5}$ (1987) consideraram $S$. santaelenae como sinônimo de $S$. sucamense; Shelley e col. ${ }^{15}$ (1984)

\footnotetext{
* Trabalho financiado parcialmente pelo Conselho Nacional de Desenvolvimento Cientifico e Tecnológico (CNPq) Processo n: 400.594/86-ZO.

- Departamento de Ecologia, Divisão de Entomologia do Instituto Nacional de Pesquisas da Amazônia, INPA/MCT Caixa Postal 478 - 69000 - Manaus, AM - Brasil.
} 
colocaram $S$. santaelenae na sinonímia de $S$. samboni Jennings, 1915; Coscarón ${ }^{1,2}$ (1984, 1987) situou $S$. sucamense no subgênero Ectemnaspis; Coscarón ${ }^{2}$ (1987) aceita $S$. santaelenae na sinonímia de $S$. samboni e a transfere para o subgênero Psilopelmia; Crosskey ${ }^{5}$ (1987) coloca $S$. samboni, $S$. sucamense e $S$. iracouboense no subgênero Psilopelmia.

Após revisar material-tipo de $S$. sucamense (série-típica, e material topotípico adicional), de $S$. samboni (parátipo e material adicional proveniente da Venezuela e do México), de $S$. iracouboense (topótipos da série-típica e grande quantidade de imaturos e adultos de $S$. iracouboense que coletei na extensa área do Escudo das Guianas: divisas territoriais entre o Brasil e Venezuela, Guiana, Suriname e Guiana Francesa), considero que tanto $S$. santaelenae como $S$. sucamense são sinônimas de $S$. iracouboense e que esta conjuntamente com $S$. samboni pertencem ao subgênero Psilopelmia.

Uma análise mais pormenorizada da diferenciação entre $S$. samboni, $S$. iracouboense e $S$. downsi (espécies muito próximas dentro do subgênero Psilopelmia) a ser publicada em futuro próximo, está sendo feita, principalmente no sentido de caracterizar os imaturos.

Material examinado: Simulium samboni Jennings, 1915 - INPA: n:: 4002 (1 fêmea em alfinete, Parátipo), Empire, Canal Zone, Panamá, A.J. Jennings. no 6200 (1 fêmea + exúvia pupal e casulo, em alfinete / 6200-1; 1 macho + exúvia pupal e casulo, em alfinete / 6200-2), Orição, Arrecife, Distrito Federal, Venezuela, col. J. Ramírez-Pérez. ISET: (3 larvas/ 2 pupas) San Vicente, Oaxaca, México, 05.III.1952, col. C. Martinez; ( 2 machos e 2 fêmeas com as respectivas exúvias pupais, no álcool), La Trinidad, Córdoba, Veracruz, México, 14. VIII.1948, col. I. Cordova. Simulium iracouboense Floch \& Abonnenc ${ }^{6}, 1946$ - IPGF (Todo o material em lâminas e procedente da Guiana Francesa): Crique Blanche, Fevr. 1946, n. 688-A (1 pupa), n.688-B (2 pupas); Cafésoca, 1.6.1946, 1 lam. (1 macho + respectiva exúvia pupal), 1 lam. (1 fêmea + respectiva exúvia pupal); Cafésoca, 5.6.1946, 1 lam. (1 fêmea + respectiva exúvia pupal), 1 lam. (1 macho + respectiva exúvia pupal); material adicional do Saut Koloboula, Maroni, 10.10.1947, 3 lâminas cada uma com um macho, 1 lam. com 5 exúvias pupais. INPA: Grande quantidade de adultos, pupas e larvas dos seguintes locais: n? 5861 - Rio Oiapoque (divisa Brasil/ Guiana Francesa), 2425.IX.1981, col. V. Py-Daniel; n? 6003 - Rio Uatumã, Amazonas, 3.XII.1983, col. V. PyDaniel \& U.C. Barbosa \& Darwich, A.; n.s
6134,6136 a $6140,6146,6148$ a 6150,6152 a 6153, Rio Trombetas, Pará (e muitas outras coletas feitas desde os seus formadores Poana e Anamú até o encontro com o Mapuera), 19851986, col. V. Py-Daniel \& U.C. Barbosa; Rio Uraricoera, Roraima (muitas coletas feitas desde os seus formadores na divisa com a Venezuela), 1987-1988, col. V. Py-Daniel \& U.C. Barbosa. Simulium sucamense Nunes de Mello, 1974 - INPA: no 5946-5 (1 fêmea em alf., Holótipo, a exúvia pupal correspondente foi perdida), 5946-4 (exúvia pupal no álcool, Alátipo, macho perdido), 5946-1 (1 fêmea e respectiva exúvia pupal em lâmina, Parátipo), 5946-17 (1 macho em alf. + respectiva exúvia pupal no álcool, Parátipo), 5946 (muitos adultos, pupas e larvas no álcool), Igarapé Surrão, Roraima, XII.1972, cols. J.A.S. Nunes de Mello \& Eduardo V. da Silva; n. 5930 (muitas larvas e pupas e um macho criado $\langle 5930-20\rangle$ ), Rio Surumu, 29.XI.1972, cols. J.A.S. Nunes de Mello \& Eduardo V. da Silva.

\section{Simulium (Hemicnetha) rubrithorax Lutz, 1909} Simulium major Lane \& Porto, 1940 syn.n.

Após comparar os tipos de $S$. major com material identificado por Lutz como S. rubrithorax (e com muito material adicional de diferentes localidades) não encontrei diferenças que possam determinar a validade de $S$. major, portanto, considero-a sinônima de $S$. rubrithorax.

Material examinado: Simulium major Lane \& Porto, 1940 - FSP 7 fêmeas em alfinetes, sendo o n. 1325 (Holótipo) e os n.s 1326 a 1330 (Parátipos), Ponce, Chapada, Mato Grosso, (600 m.), VIII-34, col. J. Lane. Simulium rubrithorax Lutz, 1909 - INPA n: 6201 (3 fêmeas em alf.), Brazil, col. A. Lutz; INPA n: 6056 (muitas larvas e pupas no álcool), 6056-1 (1 fêmea em alf. capturada picando pessoa), 6056-2 (1 macho + respectiva exúvia pupal no álcool), 6056-3A/B (2 machos em alf. + respectivas exúvias pupais no álcool), $6056-3 \mathrm{C} / \mathrm{D} / \mathrm{E} / \mathrm{F}$ (4 machos + respectivas exúvias pupais no álcool), Gróta, Mirassól, São Paulo, 4.VIII.1977, cols. V. Py-Daniel \& J.L.M. Diniz; INPA n. 6058 (1 fêmea em alf. capturada picando pessoa), Estrada entre Sobradinho a Brasília, Brasília, Distrito Federal, 21.V.1976, col. Wilson R. Lourenço; INPA n. 6180 (muitas larvas e pupas no álcool), Bairro dos Franças, Curitiba, Paraná, 4.03.1983, col. Ana Lozovei.

Simulium (Grenieriella) Vargas \& Nájera, 1951

Vargas \& Nájera' ${ }^{17}$ (1951) descreveram o sub- 
gênero Grenieriella designando como subgenotipo S. lahillei Paterson \& Shannon, 1927, e ainda incluindo no mesmo $S$. chalcocoma Knab, 1914 e S. pruinosum Lutz, 1910. Stone ${ }^{16}$ (1963) colocou este subgênero na sinonímia de Chirostilbia, mas manteve a grafia correta. Crosskey ${ }^{3}$ (1969) na tentativa de comparar os subgêneros etiópicos, Xenosimulium e Anasolen, como o subgênero neotropical Grenieriella, usou pela primeira vez a grafia errada: Grenierella.

Este erro induziu trabalhos posteriores a seguí-lo como: Crosskey ${ }^{4,5}(1981,1987)$, MaiaHerzog e col. ${ }^{9}$ (1985), Coscarón ${ }^{2}$ (1987).

Sugiro, para trabalhos futuros, que seja usada a grafia correta do subgênero, que é Grenieriella.

Simulium (Grenieriella) pruinosum Lutz, 1910, revalidada

Macquart ${ }^{8}$ (1838) descreveu Simulium nigrimanum com base num macho; sua diagnose que serve para a grande maioria dos simulídeos neotropicais e não faz referência a fêmeas.

Shelley e col..$^{15}$ (1984) pela análise de duas fêmeas depositadas no MNHN sugerem que estas poderiam ser síntipos e que a designação de um macho, no trabalho original de Macquart, poderia ser um erro tipográfico. Pela análise dessas fêmeas, Shelley e col. ${ }^{15}$ e a comparação com o lectóptico de Simulium pruinosum Lutz, 1910, depositado no IOC, decidiram que Simulium pruinosum deveria ser sinônima de $S$. nigrimanum.

Examinei as duas fêmeas depositadas no MNHN e o lectóptipo fêmea de Simulium pruinosum, material o qual Shelley e col. ${ }^{15}$ basearam-se para propor a referida sinonímia e concordo que as três fêmeas pertencem à mesma espécie e denominada por Lutz (1910) como Simulium pruinosum.

Quanto à sinonímia do nome, discordo, pois com o mesmo tipo de inferência que a sinoní- mia foi proposta (erro tipográfico) pode ser dito também que: Macquart identificou erroneamente o sexo do exemplar examinado; que o macho, que fundamentou a descrição da espécie foi perdido e que posteriormente foram agregadas, por Macquart, estas duas fêmeas que Shelley e col. ${ }^{15}$ designaram síntipos.

Portanto, para não aumentar o grau de incerteza e insegurança taxionômica, proponho que Simulium pruinosum, seja revalidada e que $S i$ mulium nigrimanum Macquart, 1838, volte a ocupar a posição de species inquirendae.

Siglas usadas para o material examinado:

FSP - Faculdade de Saúde Pública, Departamento de Epidemiologia, Universidade de São Paulo, SP, Brasil.

IB - Instituto de Biomedicina, Villa de Cura, Venezuela.

INPA - Instituto Nacional de Pesquisas da Amazônia, Departamento de Entomologia, Manaus, AM, Brasil.

IOC - Instituto Oswaldo Cruz, Rio de Janeiro, RJ, Brasil.

IPGF - "Institut Pasteur de la Guyane Française", Cayenne, Guiana Francesa.

ISET - Instituto de Salubridad y Enfermedad Tropicales, México, México.

MNHN - "Muséum National d'Histoire Naturelle", Paris, França.

\section{AGRADECIMENTOS}

Aos Drs. O.P. Forattini (FSP), Y. Robin (IPGF), J. Ramírez-Pérez (IB), P.G. García (ISET), L. Matile (MNHN), M. Maia-Herzog (IOC), por permitirem acesso ou empréstimo do material estudado.

PY-DANIEL, V. [Simulium new synonymies and corrections, with resurrection of Simulium pruinosum Lutz, 1904 (Diptera, Culicomorpha, Simuliidae)]. Rev. Saúde públ., S. Paulo, 23: 254-7, 1989.

\begin{abstract}
The following synonyms are proposed: Simulium antunesi (Lane \& Porto, 1940), Simulium mauense Nunes de Mello, 1974 and Simulium nilesi Rambajam, 1979 are synonyms of Simulium perflavum Roubaud, 1906; Simulium sucamense Nunes de Mello, 1974 and Simulium santaelenae Ramirez-Pérez \& Peterson, 1981 are synonyms of Simulium (Psilopelmia) iracouboense Floch \& Abonnenc, 1946; Simulium major Lane \& Porto, 1940 are synonym of Simulium (Hemicnetha) rubrithorax Lutz, 1909. The recent use of the miswriting name Grenierella to the subgenus Grenieriella Vargas \& Nájera, 1951 is mentioned. Simulium (Grenieriella) pruinosum Lutz, 1910 is revalidated and Simulium nigrimanum Macquart, 1838 should be considered species inquirendae.
\end{abstract}

KEYWORDS: Simuliidae, classification. 


\section{REFERÊNCIAS BIBLIOGRÁFICAS}

1. COSCARÓN, S. Revisión del Subgênero Simulium (Ectemnaspis) Enderlein (Simuliidae, Diptera, Insecta). Rev. Soc. Entomol. arg., 43:283-325, 1984.

2. COSCARÓN, S. El Simulium Latreille en la región neotropical: análisis de los grupos supraespecíficos, especies que los integran y distribuición geográfica (Simuliidae, Diptera). Belém, Museu Paraense Emilio Goeldi, 1987.

3. CROSSKEY, R.W. A re-classification of the Simuliidae (Diptera) of Africa and its Islands. Bull. Brit. Mus. (N.H.) Entomol. Suppl. 14:1-195, 1969.

4. CRUSSKEY, R.W. Simuliid taxonomy: the contemporany scene. In: Laird, M. ed. Blackflies: the future for biological methods in integrated control. New York, Academic Press, 1981. p.3-18.

5 CROSSKEY, R.W. An annotated checklist of the world black flies (Diptera: Simuliidae). In: Kim Ke Chung \& Merritt, R.W., eds. Black flies (ecology, population management and annotated world list). Pensylvannia, Penn State Press, 1987. p.425-528.

6. FLOCH, H. \& ABONNENC, E. Simulidés de la Guyane Française (II) $S$. cauchense n. sp., $S$. oyapockense n.sp., S. iracouboense n. sp. Publ. Inst. Pasteur Guyane, 137:1-19, 1946.

7. LANE, J. \& PORTO, C.E. Simulideos dà região neotropical. III - Descrição de novas especies dos gêneros "Simulium" e "Eusimulium". Arq. Inst. Biol., S. Paulo, 11:189-95, 1940.

8. MACQUART, J. Diptéres exotiques nouveaux ou peu connus. Part. 1. Mem. Soc. Roy. Sci. Agric. et Arts Lille, 2:9-225, 1838.

9. MAIA-HERZOG, M.; SHELLEY, A.J.; LUNA DIAS, A.P.A. Simulium hirtipupa Lutz, 1910 (Diptera: Simuliidae): Descrição dos adultos e larvả e redescrição da pupa. Mem. Inst. Oswaldo Cruz, Rio de Janeiro, 80:483-90, 1985.

10. NUNES DE MELLO, J.A.S. Simulideos (Diptera, Nematocera) do Território Federal de Roraima (Brasil). Sorocaba, SP, 1974. [Tese de Doutorado - Pontificia Universidade Católica].

11. RAMBAJAM, I. A new species of Simulium (Simuliidae: Diptera) from the Lethen area, Rupununi District, Guyana, South America. Timchri. Guyana Museum \& Zoo., Guyana, 45:63-77, 1979.

12. 'RAMÍREZ-PÉREZ, J. Los Jejenes de Venezuela. [Apresentado ao Simpósio de Oncocercosis America, CAICET, Puerto Ayacucho, Venezuela, 1983].

13. RAMÍREZ-PÉREZ, J. Revisión de la familia Simuliidae (Diptera, Nematocera) en Venezuela. Bol. Dir. Malar. San. amb., 27:21-44, 1987.

14. RAMÍREZ-PEREZ, J, \& PETERSON, B.V. Simulium santaelenae (Diptera: Simuliidae) nueva especie de jején del estado Bolivar (Venezuela). Bol. Dir. Malar. San. amb., 22:161-4, 1981.

15. SHELLEY, A.J.; LUNA DIAS, A.P.A.; MAIA-HERZOG, M. New specific synonymy (sic.) in Neotropical Simulium s.l. (Diptera: Simuliidae). Mem. Inst. Oswaldo Cruz, Rio de Janeiro, 79:143-61, 1984.

16. STONE, A. An annoted list of genus-group names in the family Simuliidae (Diptera). Washington, D.C., US Government Printing Office, 1963. (US Dept. Agric. Techn. Bull., 1284).

17. VARGAS, L. \& DÍAZ NÁJERA, A. Notas sobre sistemática y morfologia de simulidos. Rev. Soc. Mex. Hist. Nat. 12(1/4):123-72, 1951.

Recebido para publicação em $22 / 11 / 88$. Reapresentado em 4/4/89.

Aprovado para publicação em 5/5/89. 